\title{
A New Validation Method for X-ray Mammogram Registration Algorithms Using a Projection Model of Breast X-ray Compression
}

\author{
John H. Hipwell*, Christine Tanner, William R. Crum, Julia A. Schnabel, Member, IEEE, and \\ David J. Hawkes, Member, IEEE
}

\begin{abstract}
Establishing spatial correspondence between features visible in $\mathrm{X}$-ray mammograms obtained at different times has great potential to aid assessment and quantitation of change in the breast indicative of malignancy. The literature contains numerous nonrigid registration algorithms developed for this purpose, but existing approaches are flawed by the assumption of inappropriate 2-D transformation models and quantitative estimation of registration accuracy is limited. In this paper, we describe a novel validation method which simulates plausible mammographic compressions of the breast using a magnetic resonance imaging (MRI) derived finite element model. By projecting the resulting known 3-D displacements into 2-D and generating pseudo-mammograms from these same compressed magnetic resonance (MR) volumes, we can generate convincing images with known 2-D displacements with which to validate a registration algorithm. We illustrate this approach by computing the accuracy for two conventional nonrigid 2-D registration algorithms applied to mammographic test images generated from three patient MR datasets. We show that the accuracy of these algorithms is close to the best achievable using a 2-D one-to-one correspondence model but that new algorithms incorporating more representative transformation models are required to achieve sufficiently accurate registrations for this application.
\end{abstract}

Index Terms-Biomedical X-ray imaging, image registration, mammography, modeling, validation.

\section{INTRODUCTION}

$\mathbf{I}$ $\mathrm{N}$ order to determine the presence or classification of breast cancer from X-ray mammograms, radiologists routinely compare images. This comparison may be made with mammograms obtained on a previous occasion, with alternate views of the same breast obtained during the same screening visit, or with the same view of the other breast as a means of determining any asymmetry that might be present. Clearly this comparison helps to confirm or refute the radiologist's appraisal of the disease and may enable an assessment of change and hence disease progression to be made.

Manuscript received February 9, 2007; revised June 15, 2007. This work was supported by the Medical Images and Signals IRC (EPSRC GR/N14248/01 and UK Medical Research Council under Grant D2025/31) and subsequently in collaboration with Kodak Ltd. via DTI Technology Programme under Grant TP/3/IMG/6/I/15558. Asterisk indicates corresponding author.

*J. H. Hipwell is with the University College London, Centre for Medical Image Computing, London, WC1E 6BT U.K. (e-mail: j.hipwell@ucl.ac.uk).

C. Tanner, W. R. Crum, J. A. Schnabel, and D. J. Hawkes are with the University College London, Centre for Medical Image Computing, London, WC1E 6BT U.K.

Digital Object Identifier 10.1109/TMI.2007.903569
The comparison is complicated however, by the projective nature of X-ray mammography, differences in the degree of the applied compression and the extent to which the breast may have "rolled" prior to this compression being applied. These factors may limit the accuracy with which corresponding regions of the breast can be identified, generate differences between mammograms which are falsely identified as malignant or obscure real changes in the breast tissue.

Accurate image registration increases the specificity of lesion detection algorithms [8], [15], [20], [43], [51] and has the potential to enable subtle breast density changes to be detected. The latter may offer insights into the early development of breast cancer given existing evidence that breast density is a risk factor for breast cancer [4].

There have been many methods proposed for registering X-ray mammograms: feature based approaches [13], [18], [19]; intensity based algorithms [24], [27]; and methods combining aspects of both [50]. However, they are all fundamentally flawed as they fail to take into account the complex 3-D displacements of anatomy that contribute to the changes seen on the conventional X-ray projection of the compressed breast. In other words, the derived transformations can all be expressed as a single displacement vector at each point in the target image resulting in a one-to-one correspondence between points in the registered images. This is erroneous because mammogram correspondence is actually one-to-many, with a single point in one mammogram corresponding to a locus of points in the other mammogram [11].

Quantitative validation, when performed, is most commonly limited to the error associated with matching particular lesions identified by a clinician [6], [18], [44], [45]. This approach is restricted to the region of the lesion, is dependent upon the visibility of the lesion in each view and assumes the very knowledge that the imaging is aiming to elicit. Kumar et al. [13] calculate the proportion of feature points correctly matched but apply unrepresentative known 2-D transformations. Finally, the performance of a given algorithm is frequently only assessed visually [24], [26], [50].

We propose a new validation method which uses 3-D displacements obtained from computational bio-mechanical models of the breast. Our method creates plausible mammographic compressions of the breast using a magnetic resonance imaging (MRI) derived finite element (FE) model. Images representing processed X-ray "pseudo-mammograms" are generated by projecting these FE deformed magnetic resonance 
(MR) volumes, producing test image pairs with known 2-D displacements with which to perform a registration validation. Note that by moving from 3-D to 2-D the displacement field in the projection images consists of multiple 2-D displacements defined at each pixel which are retained and used to compute the registration error.

To illustrate this approach, we compute the accuracy of nonrigid registrations of 2-D mammographic test images generated from three patient MR datasets. We evaluate and compare the performance of two registration algorithms that have previously proved accurate in 3-D MR breast registrations [5], [41]. Although it is well known that these algorithms cannot accurately represent a 3-D deformation projected into 2-D, this has not prevented many authors reporting the use of such algorithms in the literature [13], [18], [19], [24], [27], [50]. Therefore, as a prelude to future studies, we have evaluated these existing methods. We stress that the purpose of this empirical evaluation is to illustrate our novel validation technique and convince the reader that meaningful figures can be obtained. Presenting the results alongside figures for the initial misregistration and the upper bound achievable for a one-to-one 2-D transformation, indicates the insights that could be achieved were a more extensive validation to be performed.

We intend to use this validation method to aid development of new registration algorithms which recover the true spatial correspondence between the images.

\section{CONVEntional REgistration ERROR CALCULATION}

Before describing the components of our registration validation method, we consider the conventional means of estimating the registration error at a point $(u, v)$, for a one-to-one 2-D nonrigid registration.

Typically this would involve calculating the distance, $d_{e}$, between corresponding points $\mathbf{p}_{\mathbf{T}}$ and $\mathbf{p}_{\mathbf{S}}$ in the target and source images, respectively, after application of the computed registration transformation (from target to source), $T_{R}$

$$
d_{e}=\left\|T_{R}\left(\mathbf{p}_{\mathbf{T}}\right)-\mathbf{p}_{\mathbf{S}}\right\|_{2} \text {. }
$$

The set of corresponding points $\mathbf{p}_{\mathbf{T}}$ and $\mathbf{p}_{\mathbf{S}}$ must be known in advance or be derived independently of the evaluated registration algorithm.

If the ground truth transformation (from target to source) $T_{\mathrm{GT}}$ is known, then the registration error can be estimated at any point in the target image $(u, v)$ since $\mathbf{p}_{\mathbf{S}}=T_{\mathrm{GT}}\left(\mathbf{p}_{\mathbf{T}}\right)$ :

$$
d_{e}(u, v)=\left\|T_{R}(u, v)-T_{\mathrm{GT}}(u, v)\right\|_{2}
$$

For mammogram registration, however, the ground truth transformation is a continuous 3-D deformation which, when projected, cannot be represented as a one-to-one 2-D mapping between points in the target and source images. It can, however, be represented by a one-to-many 2-D mapping and this will be described in Section III-D.

\section{METHODS}

\section{A. MR Derived FE Model of Breast Compression}

At the heart of our validation method is data describing the relative displacement of breast tissue caused by compression applied during routine X-ray mammography on separate occasions. This data was obtained using a 3-D FE model of the breast, constructed from segmented MR images and implemented using the FE software package ANSYS [1].

FE methods for modelling breast deformations have previously been explored by a number of authors. Applications included predicting mechanical deformations during biopsy procedures [2]; modelling compressions similar to X-ray mammography [21], [32], [52]; registration of magnetic resonance (MR) and X-ray mammograms [29]; validating a nonrigid registration of contrast-enhanced MR mammograms [35]; testing reconstruction algorithms in elastography [31], [38], [47]; and forward modelling for elastography [48].

Our FE models consist of between 40000 and 70000 10-noded tetrahedral elements. In comparison to [2], [21], [29], [32], [52] tetrahedral elements were chosen because their flexibility enables a mesh to be generated directly from the triangulated breast surface. To improve accuracy, tetrahedrals with a quadratic shape function were employed and a relatively fine mesh was used. Badly shaped elements, that is elements exceeding an aspect ratio of 20 or an angle of $165^{\circ}$, were kept to a minimum by improving the mesh quality using ANSYS. This resulted in meshes that had at least 2.7 more elements than the voxel based mesh [52] and 8.7 times more elements than the surface based hexahedral meshes [2], [21], [29], [32], [52].

Breast compression has previously been modelled as a contact problem [32], [52] by prescribed surface displacements [2] or by employing a penalty function to prevent penetration of the breast surface into the compression plates [21]. We applied surface displacement boundary conditions, with displacements only specified in the direction perpendicular to the plates. This allows slippage along the plates to occur. Nodes adjacent to the pectoral muscle were constrained to have zero displacements as in [2], [21], [29], [32], [52]. All other nodes were allowed to move freely.

Fatty and glandular tissues were modelled as homogeneous, isotropic materials with linear elasticities of $1 \mathrm{kPa}$ and $1.5 \mathrm{kPa}$, respectively, in accordance with tests reported in [33] and [42]. Skin was not modelled but its influence will be investigated in subsequent refinement of our models. Elasticity of tumorous tissue was varied between 3.6 and $10.8 \mathrm{kPa}$ for different tumours, to model several instances from the population. Ex vivo measurements for tumours are generally stated as the mean values for all samples for the same class of tumour (e.g., DCIS, infiltrating ductal carcinoma, infiltrating lobular carcinoma). Note also that only the ratio of the individual elasticity moduli is of importance since only displacement boundary conditions were applied. The individual tissue types are modelled as homogeneous, which is of course a simplification. In comparison to previous studies, our FE configuration was selected based on the accuracy of linear, nonlinear, and hyperelastic models to predict the location of internal breast structures after a $20 \%$ in vivo compression for two volunteers [42]. This evaluation 


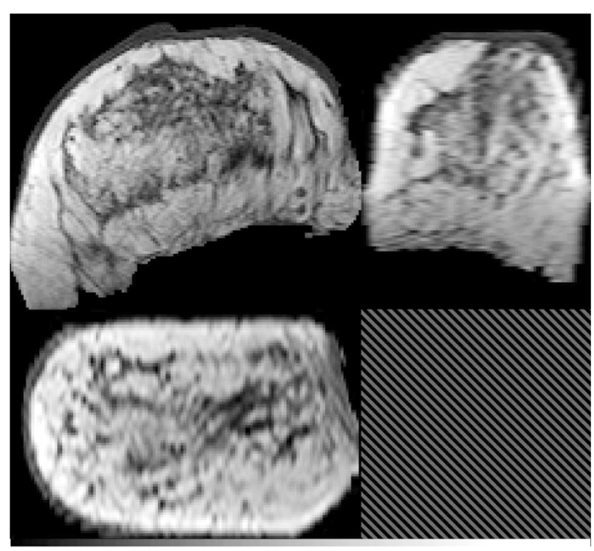

(a)

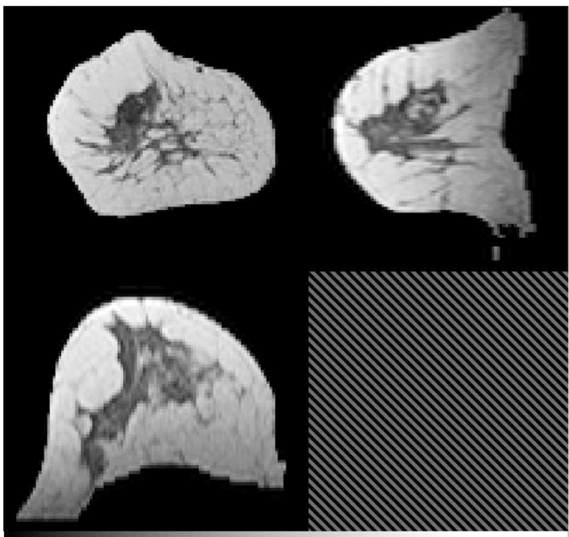

(c)

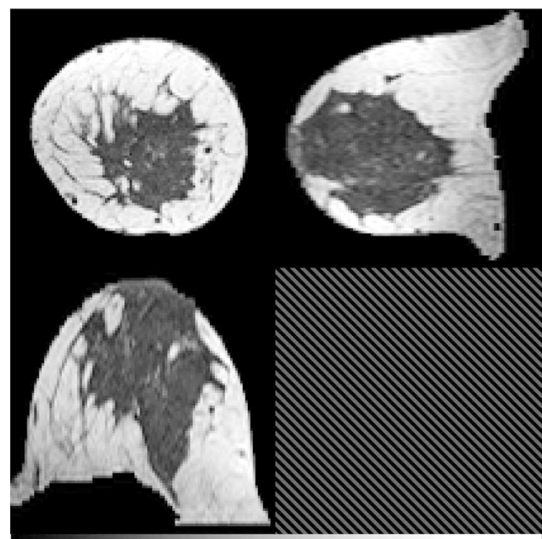

(e)

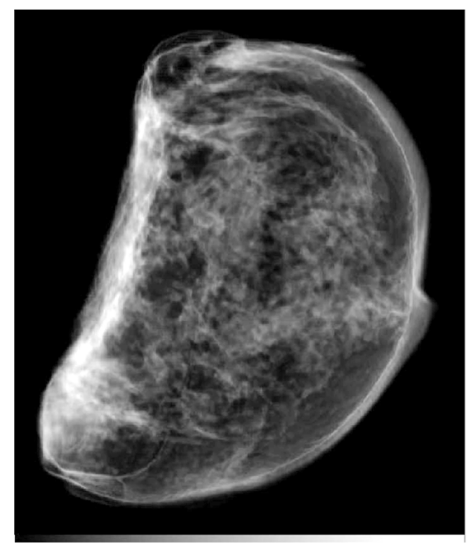

(b)

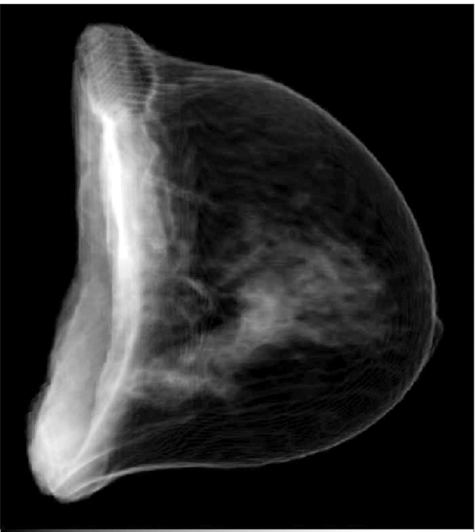

(d)

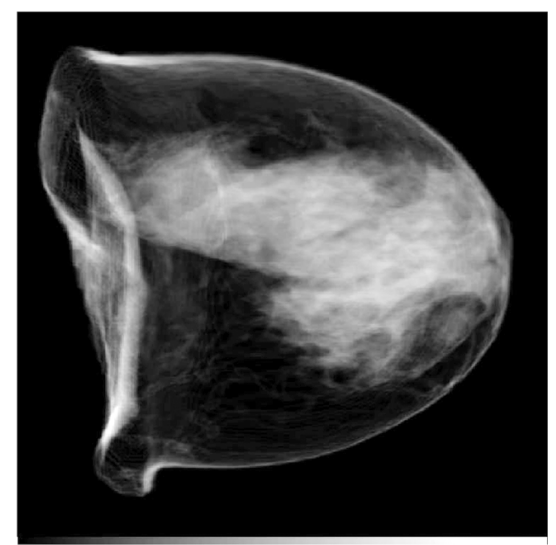

(f)

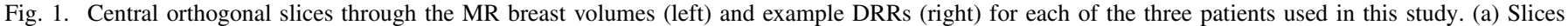

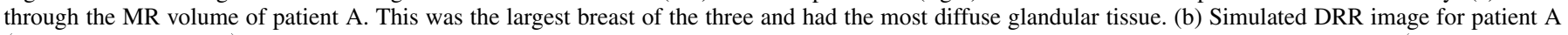
$\left(\alpha=50 \%, \beta=-10^{\circ}\right)$. (c) Slices through the MR volume of patient B, the smallest breast of the three. (d) Simulated DRR image for patient B $(\alpha=50 \%, \beta=$

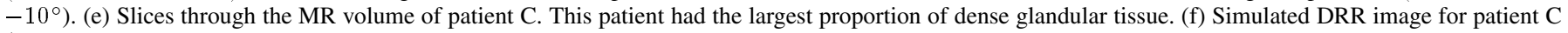
$\left(\alpha=50 \%, \beta=-10^{\circ}\right)$.

included models covering the wide range of reported ex vivo elastic properties [12], [33], [49] and modifications to them [2], [32]. Linear models performed as well as nonlinear models for these deformations and hence were selected for this work. The three tissue types (fat, glandular, and tumorous) were segmented from the MR volumes by manual thresholding (after correction for inhomogeneities [39]), and implausible regions resulting from this segmentation were removed in a subsequent manual processing step. A Poisson's ratio of 0.475 was chosen to allow for volume changes due to reduced blood volume as a result of the compression.
Cranio-caudal compressions for different patient visits were simulated by varying both the percentage compression, $\alpha$, and the angle from the cranio-caudal axis (in the coronal plane) at which this compression is applied, $\beta$. Combinations of these two parameters produced $N$ FE model deformations, $C_{i}\left(\alpha_{i}, \beta_{i}\right): i=1, \ldots, N$, and these in turn generated $N_{p q}$ multiple pairs of compression differences, $\left\{C_{p q}\left(\left[\alpha_{p}, \beta_{p}\right],\left[\alpha_{q}, \beta_{q}\right]\right): \alpha_{p} \neq \alpha_{q}\right.$ and $\left.\beta_{p} \neq \beta_{q}\right\}$, for each patient, where each difference encapsulates the relative deformation of breast tissue that might occur between mammograms acquired on two separate occasions. 


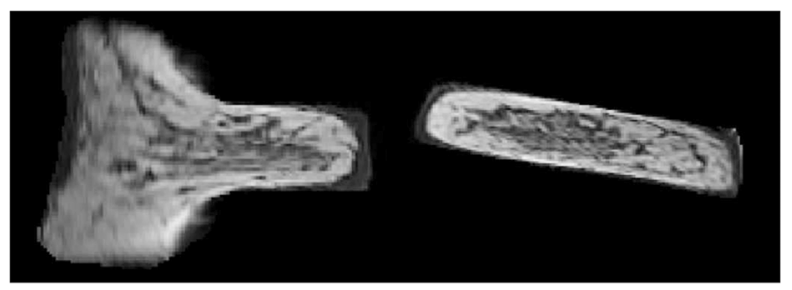

(a)

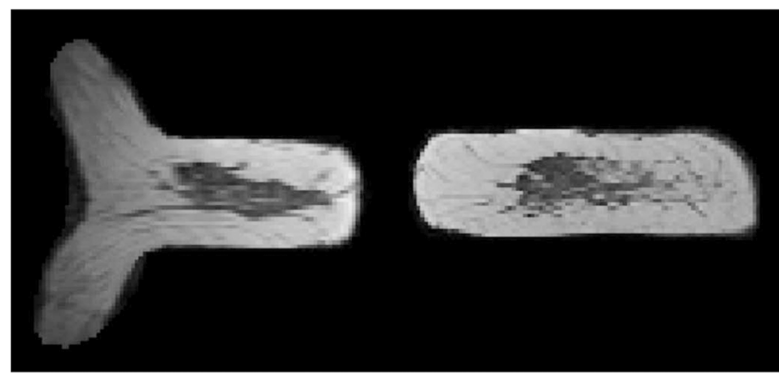

(b)

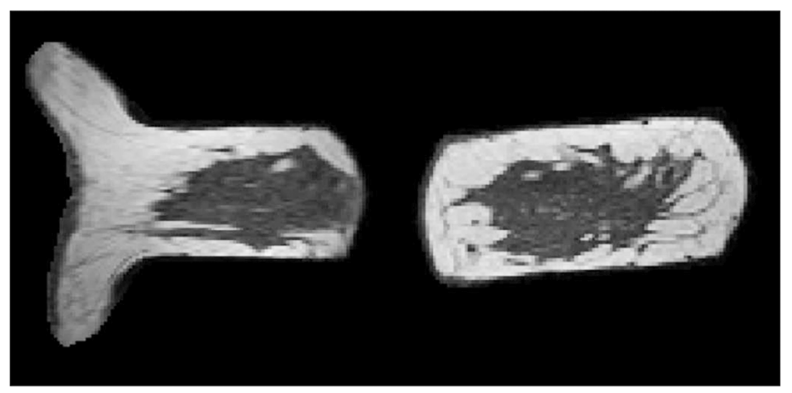

(c)

Fig. 2. Sagittal (left) and coronal (right) slices through example compressions of the three patients' MR breast volumes. (a) Patient A: $\alpha=70 \%, \beta=10^{\circ}$. (b) Patient B: $\alpha=60 \%, \beta=0^{\circ}$. (c) Patient C: $\alpha=50 \%, \beta=-5^{\circ}$.

Three patient data sets which varied substantially in both size and in the density of the glandular tissue present, were selected from a sample of patients from the MARIBS study [16] and also patients attending Guy's and St. Thomas' breast clinics (Fig. 1). Patient A's data set was acquired with a 3-D gradient echo sequence on a $1.5 \mathrm{~T}$ Philips system with $\mathrm{TR}=20 \mathrm{~ms}, \mathrm{TE}=5.2$ $\mathrm{ms}$, flip angle $=45^{\circ}$, size $256 \times 256 \times 30$ voxels $^{3}$, voxel dimensions $1.37 \times 1.37 \times 4.2 \mathrm{~mm}^{3}$, and axial slice orientation. Patients B's and C's data sets were acquired with a 3-D gradient echo sequence on a $1.5 \mathrm{~T}$ Siemens system with $\mathrm{TR}=12$ $\mathrm{ms}, \mathrm{TE}=5 \mathrm{~ms}$, flip angle $=35^{\circ}$, size $256 \times 256 \times 64$ voxels $^{3}$, voxel dimensions $1.33 \times 1.33 \times 2.5 \mathrm{~mm}^{3}$, and coronal slice orientation.

The resulting FE models (example compressions shown in Fig. 2) are approximate but deliver plausible deformations that encompass a range of displacements similar to those that would occur in clinical practice.

\section{B. 3-D to 2-D Projection}

Using a perspective ray-casting algorithm [23], both the MR volumes and their respective displacement fields can be projected into two dimensions as follows (Fig. 3).

The 3-D data set is placed at a particular location $\vec{t}$ and orientation $\vec{\theta}$ in $3-\mathrm{D}$ space (for a given patient), relative to the virtual $\mathrm{X}$-ray source position (the origin) and close to the $512 \times 512$

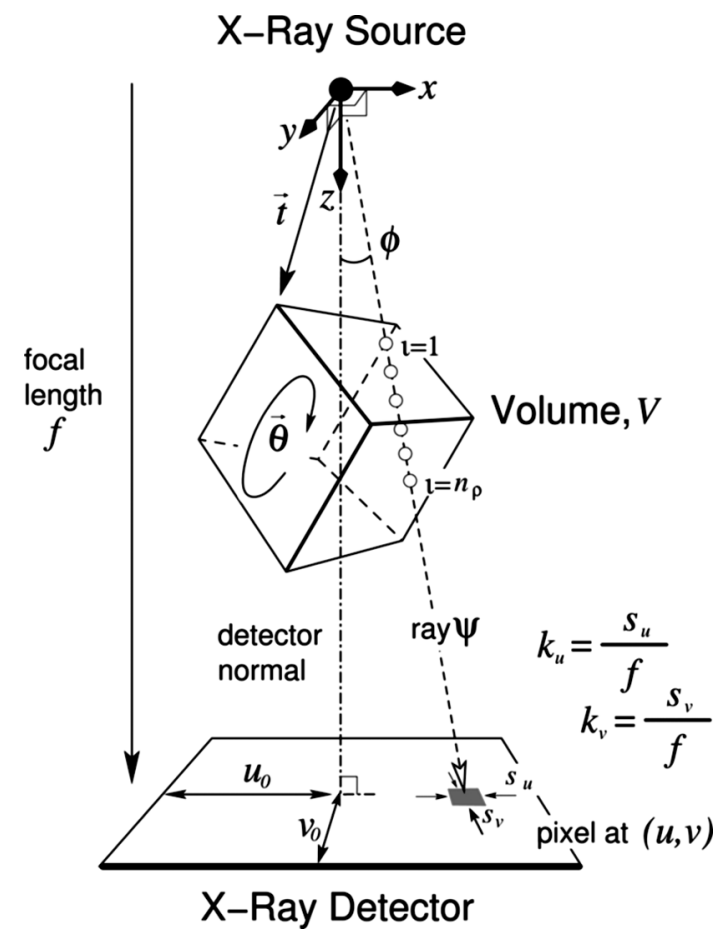

Fig. 3. Geometry for 3-D to 2-D Projection. Volume $V$ is placed at a specific location $\vec{t}$ and orientation $\vec{\theta}$ such that the projected breast comfortably fills the virtual $\mathrm{X}$-ray detector plane. The position of the detector normal $\left(u_{0}, v_{0}\right)$ together with the perspective magnification factors $k_{u}$ and $k_{v}$ define the perspective projection of the volume. Parameters $\vec{t}, \vec{\theta}, u_{0}, v_{0}, k_{u}$, and $k_{v}$ are constant for each patient. Rays $\psi$ are cast through the volume to each pixel with dimensions $s_{u} \times s_{v}$ located at $(u, v)$. The volume is interpolated at $\iota=1 \ldots n_{\rho}$ points along the ray. $\phi$ is the angle between the ray and the detector plane normal.

pixel, $0.5 \times 0.5 \mathrm{~mm}$ resolution simulated detector plane. The focal length $f$ of this virtual X-ray set was fixed at a mammographically realistic $660 \mathrm{~mm}$ (i.e., parameters $k_{u}$ and $k_{v}$, the ratios of the X-ray pixel sizes, $s_{u}$ and $s_{v}$, to the focal length, equal 1320), and for the purposes of these experiments the position of the X-ray normal from source to detector, $\left(u_{0}, v_{0}\right)$, was placed at the center of the detector plane $\left(u_{0}=v_{0}=128 \mathrm{~mm}\right)$.

The equation of a ray $\psi$ passing through the 3 -D data set from the $\mathrm{X}$-ray source to a point $(u, v)$ on the $2-\mathrm{D}$ detector plane is obtained by solving

$$
M[x y z 1]^{T}=\lambda\left[\begin{array}{lll}
u & v & 1
\end{array}\right]^{T}
$$

where $3 \times 4$ matrix

$$
M=P\left(k_{u}, k_{v}, u_{0}, v_{0}\right) T(\vec{t}) R(\vec{\theta})
$$

and the $3 \times 4$ perspective projection matrix

$$
P\left(k_{u}, k_{v}, u_{0}, v_{0}\right)=\left[\begin{array}{cccc}
k_{u} & 0 & u_{0} & 0 \\
0 & k_{v} & v_{0} & 0 \\
0 & 0 & 1 & 0
\end{array}\right],
$$

$4 \times 4$ translation matrix $T(\vec{t})$, and $4 \times 4$ rotation matrix $R(\vec{\theta})$, describe the pose and projection of the 3 -D data set relative to the detector. $\lambda$ is an arbitrary perspective magnification factor. 
To reduce the computation associated with fully 3-D raytracing, we perform bilinear interpolation at intersections of ray $\psi$ with planes of voxels aligned along a particular axis [23]. The trajectory of the ray will cause it to traverse $n_{a}$ axial, $n_{c}$ coronal and $n_{s}$ sagittal planes of voxels in some 3-D data set, $V$. The highest sampling of the ray's profile is obtained by sampling $V$ at planes $\rho$ such that the greatest number of intersections $n_{\rho}$ with the ray is obtained, i.e.,

$$
\rho=\left\{\begin{array}{llll}
\text { axial if } & n_{a} \geq n_{c} & \text { and } & n_{a} \geq n_{s} \\
\text { coronal if } & n_{c}>n_{a} & \text { and } & n_{c} \geq n_{s} \\
\text { sagittal if } & n_{s}>n_{a} & \text { and } & n_{s}>n_{c}
\end{array} .\right.
$$

The ray's profile is then given by

$$
\psi(M, u, v)=\left\{V\left(x_{\iota}, y_{\iota}, z_{\iota}\right): \iota=1 \ldots n_{\rho}\right\}
$$

where $V\left(x_{\iota}, y_{\iota}, z_{\iota}\right)$ is the value obtained from bilinearly interpolating the four voxel data values (e.g., intensity) surrounding the intersection $\left(x_{\iota}, y_{\iota}, z_{\iota}\right)$ of the ray with the $\iota$ th $\rho$ plane. This approach produces digitally reconstructed radiographs (DRRs) that are indistinguishable from those generated using fully 3-D trilinear interpolation [23]. Recent projection developments offer further reduction in processing time [3], [30].

\section{Mammographic Test Image Generation From MR}

We now describe how the 3-D to 2-D projection approach explained above enables us to generate pseudo-mammographic images from the deformed MR volumes $V_{\mathrm{MR}}$ with which to test our algorithm. These test images are intended to simulate $\mathrm{X}$-ray mammograms which have been preprocessed to remove the degrading effects of X-ray scatter and variation due to the particular X-ray exposure parameters used (mAs, kVp, etc.). Such a normalisation produces images which quantify the amount of glandular tissue present and can be obtained analytically [9], empirically by calibrating the mammography system [10], [22] or using a physical model of image acquisition [46].

Our test images are DRRs generated from the deformed MR volume. This is in contrast to the majority of DRR algorithms which have been used to simulate X-ray images from CT [7], [14], [17], [36], [37] and which exploit the similarities between these two modalities. However, MRI and X-ray are modalities which measure different physical tissue parameters (i.e., proton density, T1 and T2 versus X-ray attenuation) so some form of mapping from MR to X-ray (via a classification of breast anatomy) is required. We achieve this mapping using a manual segmentation (Section III-A) of the MR intensity $\left(I_{\mathrm{MR}}\right)$ and obtain a probability, given partial volume effects, that any intensity in the 3-D volume corresponds to glandular or tumourous breast tissue. This probability is implemented as a lookup table $L$ equal to the ratio of frequency of combined glandular and tumorous tissue $H_{G}$ to frequency of all tissue and fat, $H_{T}$ at each MR intensity, $I_{\mathrm{MR}}$ (Fig. 4)

$$
L\left(I_{\mathrm{MR}}\right)=\frac{H_{G}\left(I_{\mathrm{MR}}\right)}{H_{T}\left(I_{\mathrm{MR}}\right)}
$$

where $H_{G}$ and $H_{T}$ are the respective histograms.

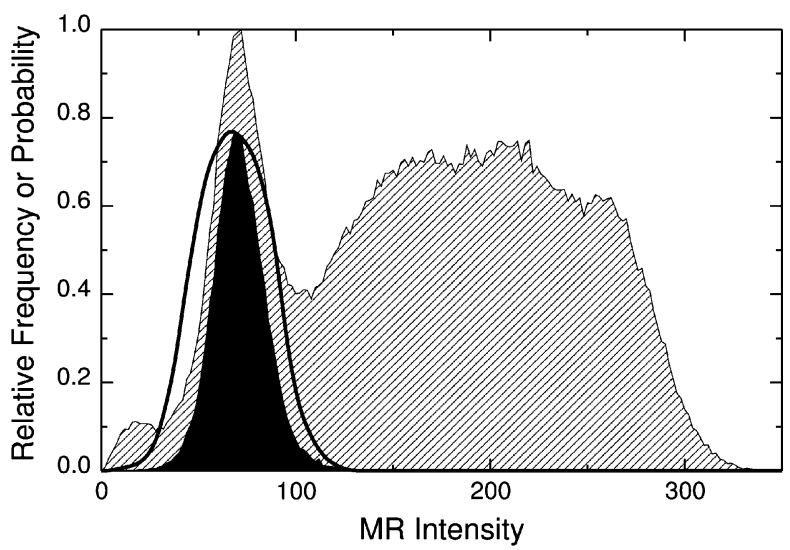

Fig. 4. Probability of glandular tissue and tumorous tissue lookup table, $L$, (indicated by the solid line) was calculated from the ratio of combined glandular and tumorous tissue frequency $H_{G}$ (dark filled histogram) to the total breast tissue frequency $H_{T}$ (light filled histogram) at each MR intensity (Section III-C). For display purposes, the two histograms have been scaled so that the total breast histogram has a maximum value of unity.

Rays were cast through the MR volume $V_{\mathrm{MR}}$ from each pixel location in the DRR $(u, v)$, as described in Section III-B. The lookup table was then used to convert the MR intensities to an estimated probability of glandular or tumorous tissue, and these values were integrated to produce the DRR intensity $D_{\mathrm{RR}}(u, v)$

$$
D_{\mathrm{RR}}(u, v)=\cos ^{2}(\phi) \sum_{\iota}^{n_{\rho}} L\left(\psi_{\mathrm{MR}}(M, u, v, \iota)\right)
$$

where $\phi$ is the angle between the ray $\psi_{\mathrm{MR}}$ and the detector plane normal, and accounts for the inverse square law reduction in intensity caused by ray's which do not hit the detector at $90^{\circ}$ (Fig. 3).

We believe these images are sufficiently mammographic-like in appearance and behavior (with respect to changes in the compression of the breast) to provide realistic images with which to quantitatively test registration algorithms (Fig. 5).

\section{Ground Truth Registration}

The 3-D relative displacement field for a given pair of compressions $C_{p}\left(\alpha_{p}, \beta_{p}\right)$ and $C_{q}\left(\alpha_{q}, \beta_{q}\right), \vec{V}_{\Delta_{p q}}$, is projected into two dimensions by treating the three orthogonal displacement components as three volumes $V_{\delta_{x}}, V_{\delta_{y}}, V_{\delta_{z}}$ and creating ray profiles using the method described in Section III-B. Corresponding points on the three component rays $\psi_{\delta_{x}}, \psi_{\delta_{y}}, \psi_{\delta_{z}}$ are then combined to create a ray profile of 3-D displacements

$$
\begin{aligned}
\psi_{\Delta_{p q}}(M, u, v)=\{ & {\left[\psi_{\delta_{x}}(M, u, v, \iota)\right.} \\
& \psi_{\delta_{y}}(M, u, v, \iota) \\
& \left.\left.\psi_{\delta_{z}}(M, u, v, \iota)\right]^{T}: \iota=1 \ldots n_{\rho}\right\}
\end{aligned}
$$

which are then projected into 2-D using the projection matrix, $P$

$$
\vec{\delta}_{\mathrm{GT}}(u, v, \iota)=P\left[\psi_{\Delta_{p q}}(M, u, v, \iota) 1\right]^{T}-[u v 1]^{T}
$$




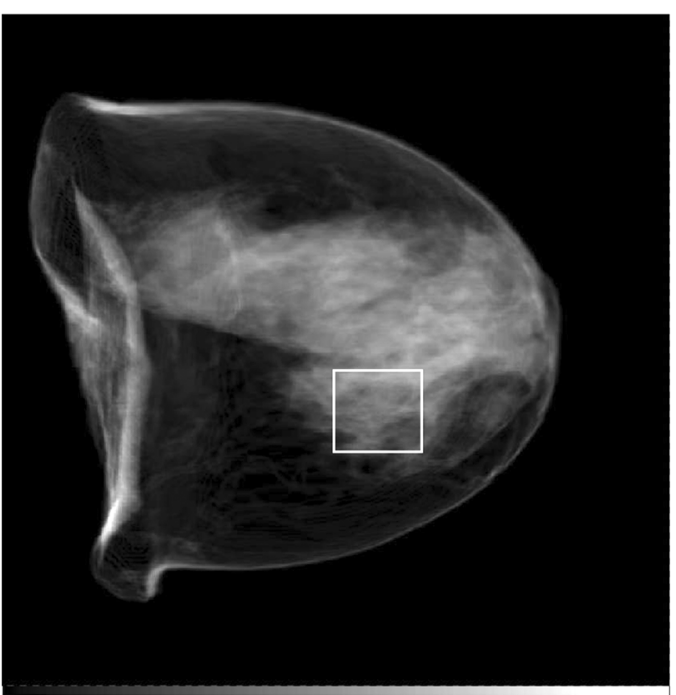

(a)

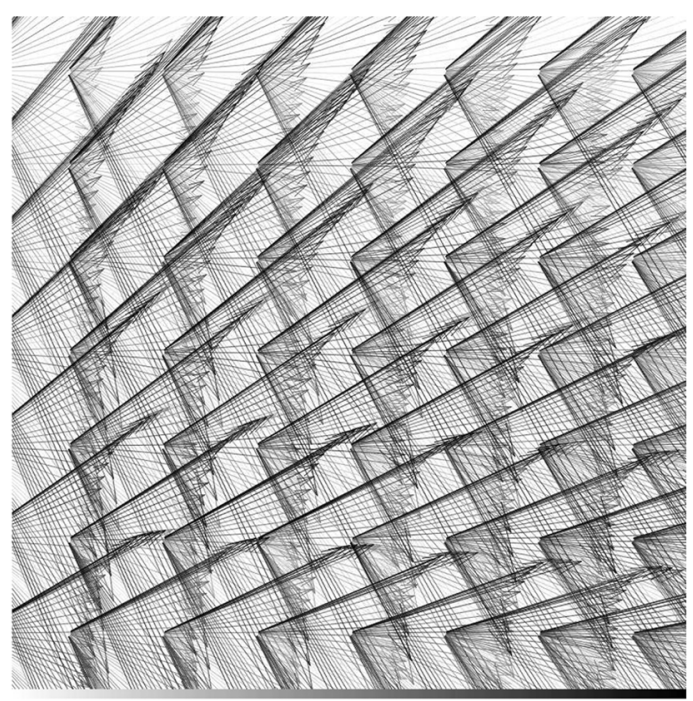

(b)

Fig. 5. Example pseudo-mammogram and projected 3-D displacement vectors for a pair of mammographic compressions illustrating the one-to-many nature of the correspondence problem. (a) Example pseudo-mammogram for patient $\mathrm{C}\left(C_{p}\left(\alpha_{p}, \beta_{p}\right): \alpha_{p}=50 \%, \beta_{p}=-10^{\circ}\right)$, with region of interest. (b) Projected 3-D displacement vectors, at regularly spaced $4 \mathrm{~mm}$ (8 pixel) locations for the region of interest in image (a), relative to a second compression $C_{q}\left(\alpha_{q}, \beta_{q}\right): \alpha_{q}=$ $70 \%, \beta_{q}=5^{\circ}$. Darker arrows indicate greater proportion of that pixel's intensity displaced.

where $\left[\begin{array}{lll}u & v & 1\end{array}\right]^{T}$ is subtracted to obtain the local displacement. This displacement is combined with the projected 3-D voxel intensities

$$
m_{\mathrm{GT}}(u, v, \iota)=\cos ^{2}(\phi) L\left(\psi_{\mathrm{MR}}(u, v, \iota)\right)
$$

using the MR volume $V_{\mathrm{MR}}$ corresponding to the first compression, $C_{p}\left(\alpha_{p}, \beta_{p}\right)$. From (11) and (12), we can generate a ground truth registration for each pixel in the source DRR, which specifies a list of intensities, $m_{\mathrm{GT}}(u, v, \iota)$, and their 2-D (i.e., projected 3-D) displacements, $\vec{\delta}_{\mathrm{GT}}(u, v, \iota)$, at each point, $(u, v)$, in the image (where $\iota=1 \ldots n_{\rho}$ ). This then is a one-to-many correspondence, consisting of a set of vector displacements plus scalar intensities which indicate the fraction of the "source" image intensity that is translated to a range of points in the "target" image. An example projected 3-D displacement field is shown in Fig. 5.

Our DRR test images are by definition in correspondence with the 3-D MR and the 3-D displacement field, so we know the ground truth deformation, the 2-D one-to-many "shipment" field, between any given pair of DRRs generated in this way. In the following section, we describe how we use both these projected 3-D displacements, $\vec{\delta}_{\mathrm{GT}}(u, v, \iota)$, and their associated intensities, $m_{\mathrm{GT}}(u, v, \iota)$, to compute a registration error which can be applied to a range of registration transformations (whether one-to-one or one-to-many).

\section{E. Registration Error Calculation}

The output of a registration algorithm can be expressed as a list of $j=1 \ldots n_{\mathrm{RG}}$ "shipments," comprising fractions of the intensity at each pixel $(u, v) \in K_{p}$ in the source image, $m_{\mathrm{RG}}(u, v, j)$, to be displaced by $\vec{\delta}_{\mathrm{RG}}(u, v, j)$, to continuous pixel coordinates in the target image. In general, for a conventional 2-D registration, a single displacement (in the opposite direction: from target to source), is obtained at each pixel in the target space (i.e., $n_{\mathrm{RG}}=1$ ) and the mass associated with this displacement (or shipment) is equal to the interpolated pixel intensity in the source image (Section II). By considering the more general case of multiple shipments at each point in the image, we allow for registration developments which produce solutions closer to the true (projected) 3-D movement of tissue in the mammogram.

For each experiment described below, we are establishing the transformation between two DRRs simulated from a pair of compressions, $C_{p}\left(\alpha_{p}, \beta_{p}\right)$ and $C_{q}\left(\alpha_{q}, \beta_{q}\right)$ (Section III-A). For each combination of $C_{p}\left(\alpha_{p}, \beta_{p}\right)$ and $C_{q}\left(\alpha_{q}, \beta_{q}\right)$, we know the ground truth registration for each pixel in the source DRR, compression $C_{p}\left(\alpha_{p}, \beta_{p}\right)$, relative to the target DRR, compression $C_{q}\left(\alpha_{q}, \beta_{q}\right)(11)$ and (12).

As illustrated in Fig. 6, the registration error at each pixel is then defined as the minimum work, $e(u, v)$, required to reconcile the shipments computed by the registration algorithm, $m_{\mathrm{RG}}$, with these ground truth shipments $m_{\mathrm{GT}}$, i.e.,

$$
e(u, v)=\min \sum_{j} \sum_{\iota} m_{e}(u, v, j, \iota) d_{e}(u, v, j, \iota)
$$

such that

$$
\begin{gathered}
m_{e}(u, v, j, 1)+m_{e}(u, v, j, 2) \\
+\cdots+m_{e}\left(u, v, j, n_{\rho}\right)=m_{\mathrm{RG}}(u, v, j) \\
\quad: j=1 \ldots n_{\mathrm{RG}} \\
m_{e}(u, v, 1, \iota)+m_{e}(u, v, 2, \iota) \quad \\
+\cdots+m_{e}\left(u, v, n_{\mathrm{RG}}, \iota\right)=m_{\mathrm{GT}}(u, v, \iota) \\
\quad: \iota=1 \ldots n_{\rho} \\
\text { and } \\
m_{e}(u, v, j, \iota) \geq 0:\left\{\iota=1 \ldots n_{\rho}, j=1 \ldots n_{\mathrm{RG}}\right\}
\end{gathered}
$$

where $d_{e}(u, v, j, \iota)$ are the Euclidean distances moved by each of the optimally reallocated intensities $m_{e}(u, v, j, \iota)$. Equation 


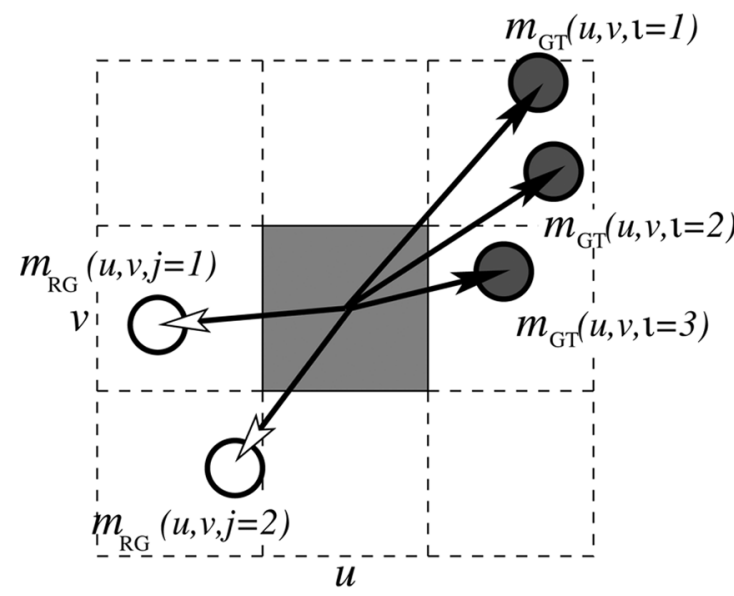

(a)

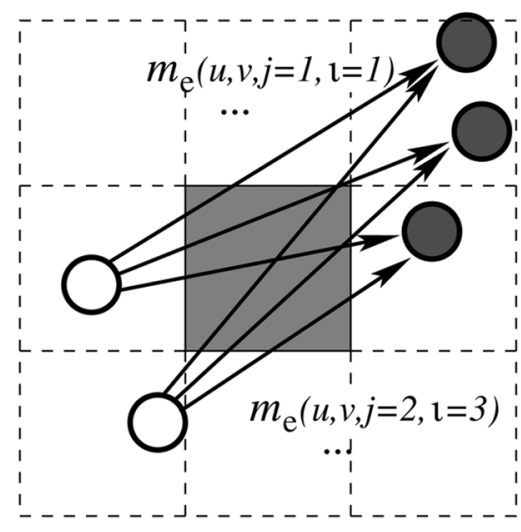

(b)

Fig. 6. Left panel: Registration displacement compared with ground-truth displacement. Right panel: Computing the registration error. (a) Output of a registration algorithm can be expressed as a list of $j=1 \ldots n_{\mathrm{RG}}$ (in this example $n_{\mathrm{RG}}=2$ ) "shipments," $m_{\mathrm{RG}}(u, v, j)$ (open circles), which specify how proportions of a given pixel's intensity are displaced from it's location at $(u, v)$. For our test images, we also have $\iota=1, \ldots, n_{\rho}$ (here $n_{\rho}=3$ ) ground truth shipments, $m_{\mathrm{GT}}(u, v, \iota)$ (closed circles), corresponding to displacements in 3-D at $n_{\rho}$ regular points along the ray projected to $(u, v)$. (b) Registration error at each pixel $(u, v)$ is equal to the minimum work $\sum m_{e}(u, v, j, \iota) d_{e}(u, v, j, \iota)$ required to redistribute the computed registration shipments $m_{\mathrm{RG}}(u, v, j)$ to coincide with the ground truth shipments $m_{\mathrm{GT}}(u, v, \iota)$, where $d_{e}(u, v, j, \iota)$ are the Euclidean distances moved by each of the optimally reallocated intensities $m_{e}(u, v, j, \iota)$.

(13) given constraints (14), (15), (16) can be solved by applying techniques from Linear Programming such as the Simplex algorithm. To obtain the mean registration error as a normalized displacement for a pair of compressions $C_{p}\left(\alpha_{p}, \beta_{p}\right), C_{q}\left(\alpha_{q}, \beta_{q}\right)$, we simply sum over all the pixels in the source image and divide by the total mass moved

$$
E_{p q}=\frac{\sum_{(u, v) \in K_{p}} e(u, v)}{\sum_{(u, v) \in K_{p}} \sum_{j} \sum_{\iota} m_{e}(u, v, j, \iota)} .
$$

$E_{p q}$ is the average shortest (Euclidean) 2-D distance that each unit of intensity in the source image has to be moved from its misregistered location in the transformed source image to its correct location in the target image (as defined by the applied 3 -D deformation). $E_{p q}$, therefore, represents the mean registration error, for glandular tissue, in millimeters.

\section{F. Nonrigid Registration Test Algorithms}

We illustrate our validation approach by measuring the accuracy of a pair of "off-the-shelf" nonrigid registration algorithms. The first is the "fluid" registration algorithm described by Crum et al. [5]. Briefly, this algorithm operates as follows. A local force driving the registration is computed at each voxel in the target image using the corresponding voxel in the source image as defined by the current deformation field. The Navier-Stokes equation for a compressible viscous fluid is solved for this driving force to yield instantaneous velocities at each point. These velocities are used to update the displacement field and the process is iterated. In 3-D applications, this algorithm performs well and the transformation model would appear to be appropriate to describe the 3-D deformations (though not projections of them) encountered in compressed breast tissue [5].

The second nonrigid registration algorithm is that of Rueckert et al. [28] which was originally developed for motion compensation in 3-D contrast enhanced (CE) MR mammography.
It is based on free-form deformations (FFDs) using $B$-splines. Local motion is modelled by manipulating an underlying mesh of $B$-spline control points. Using normalized mutual information (NMI) as a similarity measure, this method was the exemplar registration method for registration validation of CE MR mammograms using FEMs [40]. Here, we use cross correlation as a similarity measure to enable better comparison with the fluid registration method, and employ a 2-D version of the $B$-spline optimization [34].

Fig. 7 shows the results of applying these algorithms to the source and target images displayed. Visually the registrations appear to be successful: an accurate alignment of the breast edge has been obtained and overall the target and transformed source images are visually more similar than the original target and source images. This result is typical for our set of test images. There remains some residual mismatch of the internal structure of the breast in this example, however, and in attempting to eliminate this mismatch the algorithms appear to have distorted the previously well-aligned, and static, chest wall region to the left of the image.

\section{G. Minimum Registration Error of One-to-One 2-D Transformations}

The limitations inherent in computing a one-to-one 2-D correspondence between a pair of mammograms prompt the question, "What is the minimum registration error achievable with such a transformation?."

To address this issue, we also compute the registration error associated with the mean projected ground truth displacement, so that

$$
\begin{aligned}
m_{\mathrm{RG}}(u, v, j=1) & =D_{\mathrm{RR}}^{\text {source }}(u, v) \\
\vec{\delta}_{\mathrm{RG}}(u, v, j=1) & =\frac{1}{n_{\rho}} \sum_{\iota}^{n_{\rho}} \vec{\delta}_{\mathrm{GT}}(u, v, \iota) .
\end{aligned}
$$




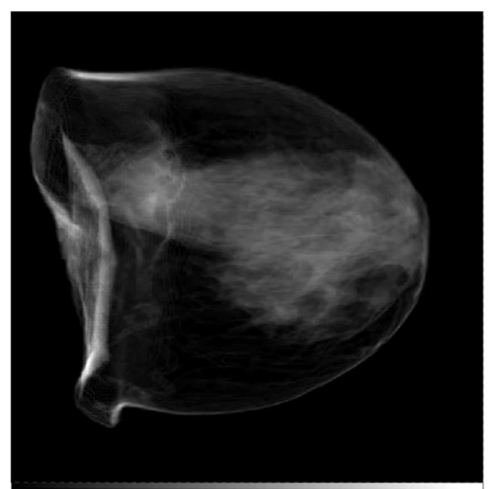

(a)

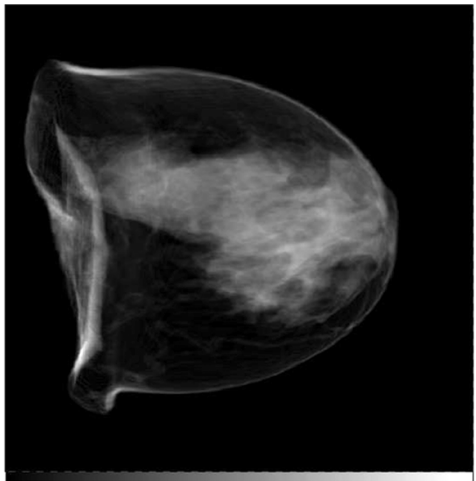

(b)

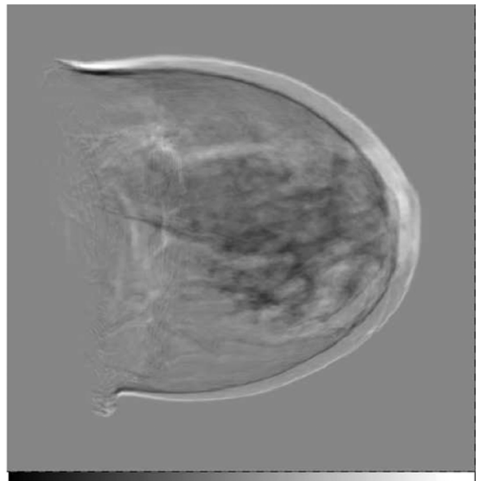

(c)

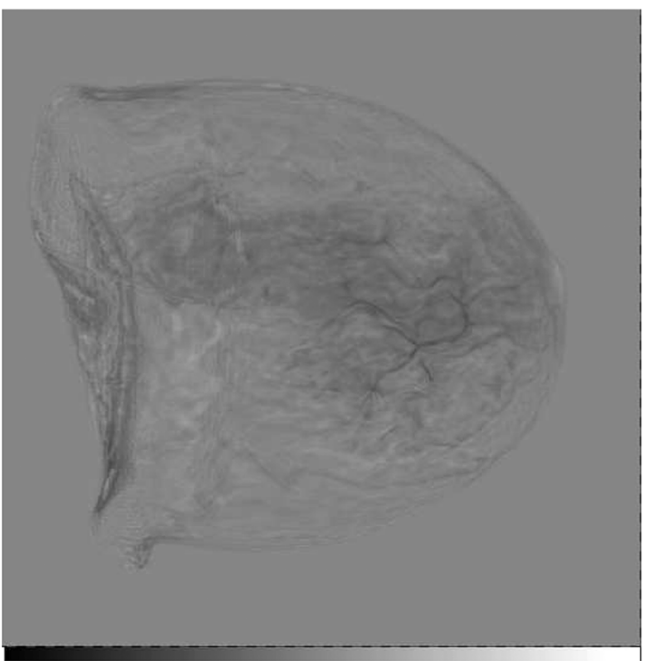

(d)

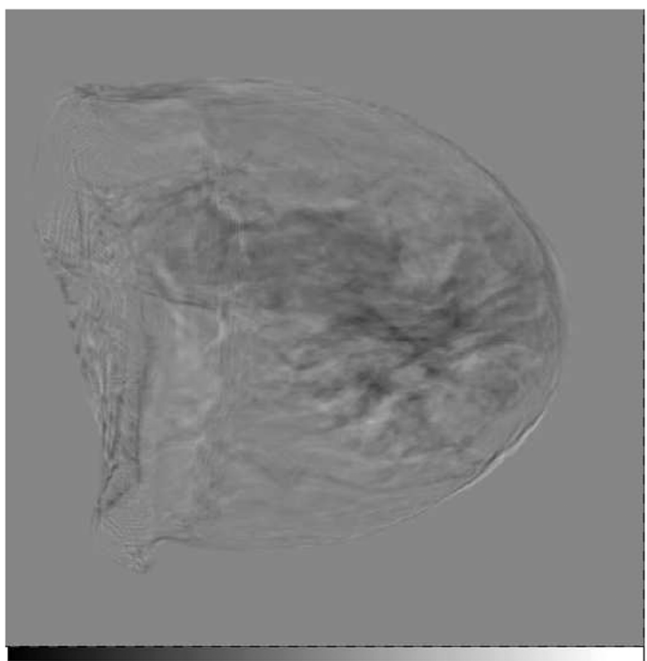

(e)

Fig. 7. Example registrations of a pair of DRRs generated from the FE deformed MRI of patient C. (a) Target pseudo-mammogram, $\alpha=70 \%, \beta=10^{\circ}$. (b) Source (floating) pseudo-mammogram to be registered to the target image, $\alpha=50 \%, \beta=-5^{\circ}$. (c) Difference between target (a) and source (b) images before registration. (d) Difference of target and transformed source image after fluid registration. (e) Difference of target and transformed source image FFD registration.

This transformation represents a "best fit" one-to-one 2-D correspondence between the source and target mammograms given the known 3-D displacements.

\section{RESULTS}

To illustrate our validation methodology, we have computed the registration error for the nonrigid registration algorithms described in Section III-F, when applied to a test set of 2-D mammographic images derived from MR, as described in Section III-C.

The test set of cranio-caudal image pairs was obtained for percentage compressions

$$
\alpha=\{50 \%, 70 \%\}
$$

and orientations

$$
\beta=\left\{-10^{\circ},-5^{\circ}, 0^{\circ}, 5^{\circ}, 10^{\circ}\right\} .
$$

The resulting mean compressed breast thickness at $70 \%$ was 35 $\mathrm{mm}$ and at $50 \%, 58 \mathrm{~mm}$, similar to the range reported in [53] and [25]. Each combination of $\alpha$ and $\beta$ produced $N=10 \mathrm{FE}$ model deformations $C(\alpha, \beta)$ and these in turn generated $N_{p q}=$

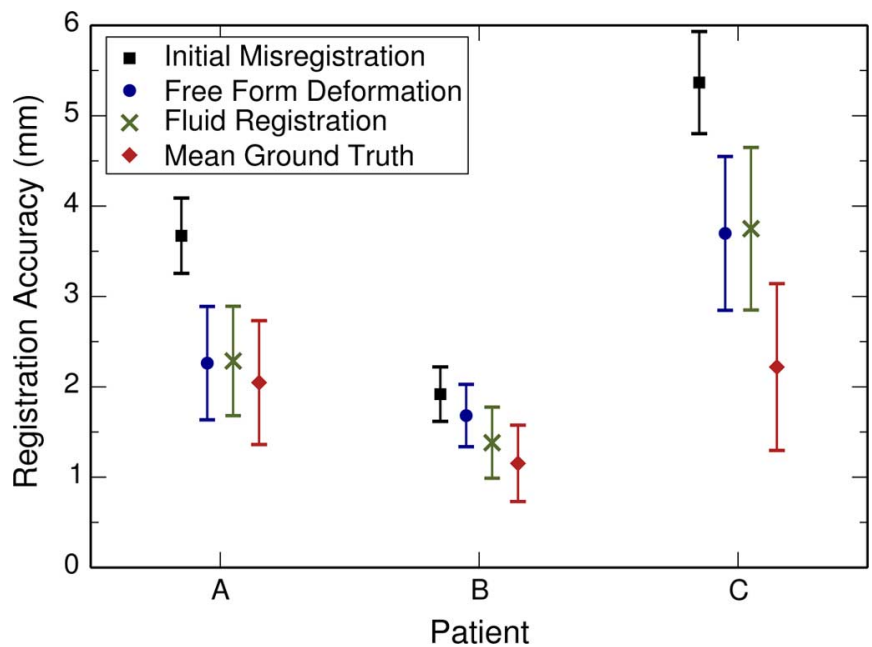

Fig. 8. Registration errors (mean and standard deviation) for each of three patients and all compression combinations.

40 pairs of compression differences, $\left(C_{p q}\left(\left[\alpha_{p}, \beta_{p}\right],\left[\alpha_{q}, \beta_{q}\right]\right)\right.$ : $\alpha_{p} \neq \alpha_{q}$ and $\beta_{p} \neq \beta_{q}$ ), for each patient.

Fig. 8 shows the mean errors for performing nonrigid registrations on these test images. The fluid registration algorithm 
registered the images to a mean accuracy of between 1.4 and 3.7 $\mathrm{mm}$, reducing the initial mean misregistrations which varied between 1.9 and $5.4 \mathrm{~mm}$. Similar performance was obtained for the FFD registrations (mean accuracies of between 1.7 and $3.7 \mathrm{~mm}$ ) using a control point spacing of $20 \mathrm{~mm}$. Refinement of these registrations using finer control point spacings (10 $\mathrm{mm}$ and $5 \mathrm{~mm}$ ) was not found to improve the accuracy. This is as expected given that a one-to-one 2-D transformation model can only expect to extract a very coarse global correspondence between mammograms. It will fail to model the local many-to-one relationship between the images despite increased degrees of freedom. In all cases however, the nonrigid registration algorithms reduced the initial misregistration.

Also given in Fig. 8 are the errors (from 1.2 to $2.2 \mathrm{~mm}$ ) obtained when the mean projected 3-D ground truth displacements, (Section III-G) are used to specify a single displacement in 2-D at each point in the source image. This is an upper bound of the minimum error the standard registration methods might hope to achieve.

\section{DISCUSSION}

The initial mean misregistration figures of 3.7 (A), 1.9 (B), and $5.4(\mathrm{C}) \mathrm{mm}$ are relatively small. This is because there is no rigid component to the misregistration, but is also a result of weighting the error by the mass of glandular tissue being displaced. This tissue tends to reside in the center of the breast and hence is displaced less than the breast edge when the compression is changed. This is particularly pronounced for patient B, which was a comparatively small breast with glandular tissue occupying a limited volume at its center. Weighting the error measurement by the mass of glandular tissue increases the sensitivity of the approach to misregistration within this region.

The trend exhibited by these initial misregistration figures (Patient $\mathrm{C}>$ Patient A > Patient B, Fig. 8) is due to differences in the area of the breast surface which can be considered to be "in contact with the compression plates." In addition, there are differences in the perspective magnification factors used for each patient to ensure that the projected breast comfortably fills the $25.6 \times 25.6 \mathrm{~cm}^{2}$ simulated detector. These two factors combine to produce larger displacements in 2-D for patient $\mathrm{C}$ and smaller displacements for patient B.

The nonrigid registration algorithms successfully reduced the misregistration present in each of the 120 test cases. The figures, however, indicate that a significant misregistration remains and this can also be seen in the residual differences between the target and transformed source images. Whilst producing a smaller intensity difference is not conclusive proof of good registration it does show that the combination of the number of degrees of freedom and the optimization procedure used by each algorithm is proving effective in making the images more similar.

An upper bound to the minimum error achievable for a one-to-one 2-D transformation is equal to the mean projected 3-D ground truth displacements. For patient A, both algorithms approached this minimum. For patients $\mathrm{B}$ and $\mathrm{C}$, they reduced the difference between the initial misregistration and this minimum by around $50 \%$.
This performance is as expected for registration algorithms which compute a 2-D transformation. Continuous diffeomorphic 2-D transformations, such as those generated by the fluid registration, imply a one-to-one correspondence between points in the source and target images. Clearly this is not the case for X-ray mammograms due to the perspective projection of the variably compressed 3-D breast. Thus, the minimum error achievable using this class of transformations (approximated by the mean projected 3-D ground truth displacements), still equates to a significant residual that cannot be surpassed. In this respect, the free-form $B$-spline registration might be considered to have an advantage in that nondiffeomorphic "folding" of the deformation can occur. Such a discontinuity of the 2-D transformation bears little resemblance to the set of projected 3-D breast deformations, however, and so cannot be expected to improve the registration. On the contrary, it is conceivable that a simpler diffeomorphic 2-D transformation might in fact be a better approximation in this respect, helping to constrain the registration to a more accurate solution.

For physically realistic results, however, new registration algorithms are required which produce nondiffeomorphic transformations that can capture the point-locus nature of the correspondence problem (the "multiple shipments," $n_{\mathrm{RG}}>1$, of Section III-E). We are developing such an algorithm and will be able to use the same approach described here to validate the resulting program. It is necessary to develop such an evaluation strategy if we are to judge whether or not a particular algorithm is improving spatial correspondence.

\section{CONCLUSION}

This paper describes a novel validation technique for X-ray mammogram registration. Our approach uses real MR breast images from which pseudo-mammograms are generated. Choosing a variety of different breast anatomies as seen in MR ensures that population variation is represented in the resulting cohort of pseudo-mammograms. By applying known compressions at a range of orientations, we reproduce plausible deformations of the breast which might have occurred during mammography on separate occasions. Projections of these known deformations can then be used to compute the accuracy of a registration algorithm. We illustrate this approach by applying it to the results of a pair of off-the-shelf nonrigid registration algorithms that have proved accurate for 3-D breast MR registrations. These are intensity based registration algorithms but the validation methodology is equally applicable to other, including feature based, algorithms. The only requirement of candidate algorithms is that they generate a transformation which can be interpreted as a set of one-to-one or one-to-many correspondences between specific points in the images. Parametric, nonparametric and feature-based registration algorithms all produce transformations which can be expressed in this way and, therefore, compared using the validation method. Error statistics can also be computed over specific regions in the images to study the ability of the algorithms to match particular image features.

It is our intention to use this validation technique to develop new registration algorithms which will be able to distinguish 3-D movement of tissue between two X-ray mammograms from 
changes in the mass of glandular tissue. This will require an extension of the method (modelling and validation) to account for the change in mass of glandular tissue.

Finally, we are developing more sophisticated computational biomechanical models of the breast and will incorporate these as they are validated. We emphasize that for the purposes of this validation methodology we only need our models to deliver the range of likely displacements encountered in clinical practice. We are not attempting to predict the precise displacement that occurs in a particular procedure. Our method does not depend upon this information.

\section{ACKNOWLEDGMENT}

The authors would like to thank the MARIBS study [16] and Guy's and St. Thomas' NHS Foundation Trust for providing the image data used in this study.

\section{REFERENCES}

[1] ANSYS. ANSYS Inc., Canonsburg, PA, 2002 [Online]. Available: http://www.ansys.com

[2] F. S. Azar, D. N. Metaxas, and M. D. Schnall, "A finite model of the breast for predicting mechanical deformations during biopsy procedure," in IEEE Workshop Math. Methods Biomed. Image Anal., 2000, pp. $38-45$

[3] W. Birkfellner, R. Seemann, M. Figl, J. Hummel, C. Ede, P. Homolka, X. Yang, P. Niederer, and H. Bergmann, "Fast DRR generation for 2-D/3-D registration," Med. Image Computing Computer-Assisted Intervention (MICCAI), vol. 3750, pt. 2, pp. 960-967, 2005.

[4] N. F. Boyd, J. W. Byng, R. A. Jong, E. K. Fishell, L. E. Little, A. B. Miller, G. A. Lockwood, D. L. Tritchler, and M. J. Yaffe, "Quantitative classification of mammographic densities and breast-cancer risk-Results from the Canadian national breast screening study," J. Nat. Cancer Inst., vol. 87, pp. 670-675, 1995.

[5] W. R. Crum, C. Tanner, and D. J. Hawkes, "Anisotropic multiscale fluid registration: Evaluation in magnetic resonance breast imaging," Phys. Med. Biol., vol. 50, pp. 5153-5174, 2005.

[6] P. Filev, L. Hadjiiski, B. Sahiner, H. P. Chan, and M. A. Helvie, "Comparison of similarity measures for the task of template matching of masses on serial mammograms," Med. Phys., vol. 32, pp. 515-529, 2005.

[7] L. M. G. Brown and T. E. Boult, "Registration of planar film radiographs with computed tomography," in Proc. MMBIA, 1996, pp. 42-51.

[8] L. Hadjiiski, B. Sahiner, H. P. Chan, N. Petrick, M. A. Helvie, and M. Gurcan, "Analysis of temporal changes of mammographic features: Computer-aided classification of malignant and benign breast masses," Med. Phys., vol. 28, pp. 2309-2317, 2001.

[9] R. Highnam and M. Brady, Mammographic Image Analysis. Norwell, MA: Kluwer, 1999.

[10] J. Kaufhold, J. A. Thomas, J. W. Eberhard, C. E. Galbo, and D. E. G. Trotter, "A calibration approach to glandular tissue composition estimation in digital mammography," Med. Phys., vol. 29, pp. 1867-1880, 2002.

[11] Y. Kita, R. Highnam, and M. Brady, "Correspondence between different view breast $\mathrm{X}$ rays using curved epipolar lines," Comput. Vis. Image Und., vol. 83, pp. 38-56, 2001.

[12] T. A. Krouskop, T. M. Wheeler, F. Kallel, B. S. Garra, and T. Hall, "Elastic moduli of breast and prostate tissues under compression," $\mathrm{Ul}$ trasonic Imag., vol. 20, pp. 260-274, 1998.

[13] S. Kumar, M. Sallam, and D. Goldgof, "Matching point features under small nonrigid motion," Pattern Recognit., vol. 34, pp. 2353-2365, 2001.

[14] P. Lacroute and M. Levoy, "Fast volume rendering using a shear-warp factorization of the viewing transformation," in Proc. SIGGRAPH, 1994, pp. 451-458.

[15] T. K. Lau and W. F. Bischof, "Automated detection of breast-tumors using the asymmetry approach," Computers Biomed. Res., vol. 24, pp. 273-295, 1991.
[16] M. O. Leach, C. R. M. Boggis, A. K. Dixon, D. F. Easton, R. A. Eeles, D. G. R. Evans, F. F. Gilbert, I. Griebsch, R. J. C. Hoff, P. Kessar, S. R. Lakhani, S. M. Moss, A. Nerurkar, A. R. Padhani, L. J. Pointon, D. Thompson, and R. M. L. Warren, "Screening with magnetic resonance imaging and mammography of a UK population at high familial risk of breast cancer: A prospective multicentre cohort study (MARIBS)," Lancet, vol. 365, pp. 1769-1778, 2005.

[17] L. Lemieux, R. Jagoe, D. R. Fish, N. D. Kitchen, and D. G. T. Thomas, "A patient-to-computed-tomography image registration method based on digitally reconstructed radiographs," Med. Phys., vol. 21, pp. 1749-1760, 1994.

[18] K. Marias, C. Behrenbruch, S. Parbhoo, A. Seifalian, and M. Brady, "A registration framework for the comparison of mammogram sequences," IEEE Trans. Med. Imag., vol. 24, no. 6, pp. 782-790, Jun. 2005.

[19] R. Marti, R. Zwiggelaar, and C. M. E. Rubin, "Automatic point correspondence and registration based on linear structures," Int. J. Pattern Recognit., vol. 16, pp. 331-340, 2002.

[20] S. Paquerault, N. Petrick, H. P. Chan, B. Sahiner, and M. A. Helvie, "Improvement of computerized mass detection on mammograms: Fusion of two-view information," Med. Phys., vol. 29, pp. 238-247, 2002.

[21] P. Pathmanathan, D. Gavaghan, J. Whiteley, M. Brady, M. Nash, P. Nielsen, and V. Rajagopal, "Predicting tumour location by simulating large deformations of the breast using a 3-D finite element model and nonlinear elasticity," in Proc. Med. Image Computing Computer-Assisted Intervention (MICCAI), 2004, vol. 3217, pp. 217-224.

[22] O. Pawluczyk, B. J. Augustine, M. J. Yaffe, D. Rico, J. W. Yang, G. E. Mawdsley, and N. F. Boyd, "A volumetric method for estimation of breast density on digitized screen-film mammograms," Med. Phys., vol. 30, pp. 352-364, 2003.

[23] G. P. Penney, "Registration of tomographic images to X-ray projections for use in image guided interventions," Ph.D. dissertation, Univ. London, London, U.K., 1999.

[24] S. Periaswamy and H. Farid, "Elastic registration in the presence of intensity variations," IEEE Trans. Med. Imag., vol. 22, no. 7, pp. 865-874, Jul. 2003.

[25] A. Poulos, D. McLean, M. Rickard, and R. Heard, "Breast compression in mammography: How much is enough?," Australas. Radiol., vol. 47, pp. 121-126, 2003.

[26] F. Richard and L. Cohen, "A new image registration technique with free boundary constraints: Application to mammography," Proc. Comput. Vis. (ECCV), vol. 2353, pt. IV, pp. 531-545, 2002

[27] F. J. P. Richard and L. D. Cohen, "A new image registration technique with free boundary constraints: Application to mammography," Comput. Vis. Image Und., vol. 89, pp. 166-196, 2003.

[28] D. Rueckert, L. I. Sonoda, C. Hayes, D. L. G. Hill, M. O. Leach, and D. J. Hawkes, "Nonrigid registration using free-form deformations: Application to breast MR images," IEEE Trans. Med. Imag., vol. 18, no. 8, pp. 712-721, Aug. 1999.

[29] N. V. Ruiter, "Registration of X-ray mammograms and MR-volumes of the female breast based on simulated mammographic deformation," Ph.D. dissertation, Univ. Mannheim, Mannheim, Germany, 2003.

[30] D. B. Russakoff, T. Rohlfing, K. Mori, D. Rueckert, A. Ho, J. R. Adler and C. R. Maurer, "Fast generation of digitally reconstructed radiographs using attenuation fields with application to 2-D-3-D image registration," IEEE Trans. Med. Imag., vol. 24, no. 11, pp. 1441-1454, Nov. 2005.

[31] A. Samani, J. Bishop, and D. B. Plewes, "A constrained modulus reconstruction technique for breast cancer assessment," IEEE Trans. Med. Imag., vol. 20, no. 9, pp. 877-885, Sep. 2001.

[32] A. Samani, J. Bishop, M. J. Yaffe, and D. B. Plewes, "Biomechanical 3-D finite element modelling of the human breast using MRI data," IEEE Trans. Med. Imag., vol. 20, no. 4, pp. 271-279, Apr. 2001

[33] A. Sarvazyan, D. Goukassian, E. Maevsky, and G. Oranskaja, "Elastic imaging as a new modality of medical imaging for cancer detection," in Proc. Int. Workshop Interaction Ultrasound Biological Media, Valenciennes, France, 1994, pp. 69-81.

[34] J. A. Schnabel, D. Rueckert, M. Quist, J. M. Blackall, A. D. CastellanoSmith, T. Hartkens, G. P. Penney, W. A. Hall, H. Liu, C. L. Truwit, F. A. Gerritsen, D. L. G. Hill, and D. J. Hawkes, , W. J. Niessen and M. A. Viergever, Eds., "A generic framework for non-rigid registration based on non-uniform multi-level free-form deformations," in Proceedings of the 4th International Conference on Medical Image Computing and Computer-Assisted Intervention. New York: Springer Verlag, 2001, Lecture Notes in Computer Science, pp. 573-581. 
[35] J. A. Schnabel, C. Tanner, A. D. Castellano-Smith, A. Degenhard, M. O. Leach, D. R. Hose, D. L. G. Hill, and D. J. Hawkes, "Validation of nonrigid image registration using finite-Element methods: Application to breast MR images," IEEE Trans. Med. Imag., vol. 22, no. 2, pp. 238-247, Feb. 2003.

[36] G. W. Sherouse, K. Novins, and E. L. Chaney, "Computation of digitally reconstructed radiographs for use in radiotherapy treatment design," Int. J. Radiation Oncol., vol. 18, pp. 651-658, 1990.

[37] R. L. Siddon, "Fast calculation of the exact radiological path for a 3-dimensional CT array," Med. Phys., vol. 12, pp. 252-255, 1985.

[38] R. Sinkus, J. Lorenzen, D. Schrader, M. Lorenzen, M. Dargatz, and D. Holz, "High-resolution tensor MR elastography for breast tumour detection," Phys. Med. Biol., vol. 45, pp. 1649-1664, 2000.

[39] J. G. Sled, A. P. Zijdenbos, and A. C. Evans, "A nonparametric method for automatic correction of intensity nonuniformity in MRI data," IEEE Trans. Med. Imag., vol. 17, no. 1, pp. 87-97, Feb. 1998.

[40] C. Tanner, J. A. Schnabel, A. Degenhard, A. D. Castellano-Smith, C. Hayes, M. O. Leach, D. R. Hose, D. L. G. Hill, and D. J. Hawkes, "Validation of volume-preserving non-rigid registration: Application to contrast-enhanced MR-mammography," in Proc. Med. Image Computing Computer-Assisted Intervention (MICCAI'02) , 2002, vol. 2488, pp. 307-314.

[41] C. Tanner, J. A. Schnabel, A. Degenhard, M. A. Hall-Craggs, S. I. Usiskin, M. O. Leach, D. R. Hose, D. L. G. Hill, and D. J. Hawkes, "Quantitative evaluation of free-form deformation registration for dynamic contrast-enhanced MR mammography," Med. Phys., vol. 34, no. 4, pp. 1221-1233, 2007.

[42] C. Tanner, J. A. Schnabel, M. O. Leach, D. R. Hose, D. L. G. Hill, and D. J. Hawkes, "Factors influencing the accuracy of biomechanical breast models," Med. Phys., vol. 33, no. 6, pp. 1758-1769, 2006.

[43] S. Timp and N. Karssemeijer, "Interval change analysis to improve computer aided detection in mammography," Med. Image Anal., vol. 10 , pp. 82-95, 2006.
[44] S. Timp, S. van Engeland, and N. Karssemeijer, "A regional registration method to find corresponding mass lesions in temporal mammogram pairs," Med. Phys., vol. 32, pp. 2629-2638, 2005.

[45] S. van Engeland, P. Snoeren, J. Hendriks, and N. Karssemeijer, "A comparison of methods for mammogram registration," IEEE Trans. Med. Imag., vol. 22, no. 11, pp. 1436-1444, Nov. 2003.

[46] S. van Engeland, P. R. Snoeren, H. Huisman, C. Boetes, and N. Karssemeijer, "Volumetric breast density estimation from full-field digital mammograms," IEEE Trans. Med. Imag., vol. 25, no. 3, pp. 273-282, Mar. 2006.

[47] E. E. W. van Houten, M. I. Miga, J. B. Weaver, F. E. Kennedy, and K. D. Paulsen, "Three-dimensional subzone-based reconstruction algorithm for MR elastography," Magnet. Reson. Med., vol. 45, pp. 827-837, 2001.

[48] C. W. Washington and M. I. Miga, "Modality independent elastography (MIE): A new approach to elasticity imaging," IEEE Trans. Med. Imag., vol. 23, no. 9, pp. 1117-1128, Sep. 2004.

[49] P. S. Wellman, "Tactile imaging," Ph.D. dissertation, Harvard Univ., Cambridge, MA, 1999.

[50] M. A. Wirth, J. Narhan, and D. Gray, "Nonrigid mammogram registration using mutual information," Proc. SPIE Med. Imag. 2002: Image Process., vol. 4684, pp. 562-573, 2002.

[51] F. F. Yin, M. L. Giger, K. Doi, C. E. Metz, C. J. Vyborny, and R. A. Schmidt, "Computerized detection of masses in digital mammograms-Analysis of bilateral subtraction images," Med. Phys., vol. 18, pp. 955-963, 1991.

[52] H. M. Yin, L. Z. Sun, G. Wang, T. Yamada, J. Wang, and M. W. Vannier, "ImageParser: A tool for finite element generation from three-dimensional medical images," BioMed. Eng. OnLine, vol. 3, no. 31, Oct. 2004.

[53] K. C. Young and A. Burch, "Radiation doses received in the UK breast screening programme in 1997 and 1998," Brit. J. Radiol., vol. 73, pp. 278-287, 2000. 